\title{
Sensor Networks - Localization and Topology
}

\author{
Marios Sfendourakis Rajagopal Nilavalan Emmanuel Antonidakis
}

\begin{abstract}
This paper examines various types of networks their characteristics and parameters. It is also looks in current ongoing research on the field of localization and positioning and work related with localization that doesn't take into account the parameter of distance between SRs. Another type of localization is also commented which is the localization in Telemetry. In addition, issues of network coverage and topology are analyzed. Network hole problems are also examined as they are of high importance when a network has to perform accurately and with a minor number of areas that are problematic or may degrade it's performance. It is easily understood that a network's overall performance is affected by a combination of attributes that need to be examined on a case by case basis.
\end{abstract}

Keywords - Fixed Stations Network, Mobile Sensors, Network, Localization, Telemetry, Network Topology, Network Holes.

\section{INTRODUCTION}

The problem of localization is under research recent years and as the applications of Sensors Networks are spread year by year in many fields, ways to find the position of a Sensor or a Transmitter with high accuracy are still tested and remain of high importance. One of the localization techniques is the process of Triangulation which was analyzed in [1]. It was also shown that in a large network with a great number of Sensors - SRs there are cases of pseudo Transmitters PTRNs which need to be examined on a case by case basis. The concept of this paper is to shed light on the various issues that should be put in consideration when a SN (Sensors Network ) has to work as an automated system. Various characteristics will be analyzed and it will be shown what makes a sensors network unique.

\section{Sensor Networks}

There are many types of Sensor Networks which are used recently in a wide area of fields. Networks are used in agriculture, for environmental monitoring, for scientific or military purposes etc. A sensor network could be described as a group of sensor nodes which co-ordinate to perform specific actions. Unlike traditional networks, sensor networks depend on dense deployment and co-ordination to carry out their tasks. But depending on the purpose that a Network is designed its architecture, topology, scalability, sensors characteristics and many other properties might vary.

\subsection{Sensor Networks types}

There are various types of Networks depending on their state, if they are mobile or not, if they are wireless or not etc. , and the analysis of all the various types of networks is beyond the scope of this paper. The networks also can be classified in accordance with the types of sensors, their topologies and their scale. When a network is spread in a large area, issues of scalability arise in combination with cases of coverage. Recent days and as sensors properties are increased, the dynamic of a network might be adapted to the demands of the purpose for which it is designed. For that reason, every network which is designed for a single purpose and working properly, could be completely unsuitable in another case. That means that every network is unique and has its own purpose and properties.

\subsubsection{Fixed Sensor Network}

In a fixed station network the sensors positions are fixed , meaning that their position is already known. Sensors positions have been acquired with GPS and the system is aware for their coordinates. In this case the network doesn't have to find the sensors position. The set of sensors are deployed within a predetermined geographical area to gather and aggregate data. The data are forwarded to a base station for processing or a remote database.

\subsubsection{Wireless Sensor Network}

A wireless sensor network (WSN) is a special kind of ad hoc network that consists of a number of sensors spread across a geographical area.

[11000]. Mobility has relegated the traditional wired network as similarly was happened with the mobile telephone to its contemporary land-line phone. Easy and simple network set up procedures allow everyone to connect to any network which is available with adequate signal. There are three basic components of a wireless LAN. The access point, which bridges the gap between the client and the Internet, the Wireless Medium and the Client/Station [2]. A typical client can be a laptop, a tablet an I -Pad, or even a smart phone with Wi-Fi. Two important aspects of the communication in wireless sensor networks are the topologies and the internal architecture. The selection of best topology in accordance with the internal architecture of the wireless sensor node is very critical job so that proper communication can be there in between all the nodes according to the application [3]. In addition to that and assuming that a base station is needed for collecting and processing the sensors network data, the positioning and placement of the base station is critical in order to optimize the total energy consumption of the network [4].

A wireless ad hoc network (WANET) is a decentralized type of wireless network. The network is ad hoc because it does not rely on a pre-existing infrastructure, such as routers in wired networks or access points in managed (infrastructure) wireless networks. Instead, each node participates in routing by forwarding data for other nodes, so the determination of which nodes forward data is made dynamically on the basis of network connectivity [5].

\subsubsection{Mobile Network}

In a mobile network the SRs doesn't have fixed positions and they are movable. The sensors might be moved in an area of interest, or distributed in a place to cover holes in one area that needs a high level coverage. In [6], it was shown that this approach has the advantage that it does not require centralized control, communication, and for that reason scale to very large networks. Flocchini et al. [7] studied a ring type self-deployment of mobile sensors in a purely decentralized and distributed fashion. In a finite time a state of static equilibrium is reached in which the sensors network uniformly cover the environment. For a mobile network the issues of coverage and energy consumption are of prime importance as the sensors move in a geographical area. Teng et al. [8] used a sensor relocation application and showed that after a sensor node failure where a coverage hole is created, a mobile sensor node can be relocated to cover the hole in a timely and energy - efficient way. 


\subsubsection{Hybrid Network}

In a Hybrid network there is a group of sensors that have fixed positions and a group of sensors that are movable. By moving the available mobile sensors, Wang et al. [9] is showing the single coverage problem in contrast with a hybrid network which is able to heal coverage holes by using the movable sensors. They used Voronoi diagrams to detect coverage holes and used one of three algorithms to calculate the target locations of sensors if holes exist. They showed the effectiveness of the protocols and calculation of algorithms under different application requirements and working conditions.

\subsection{Sensors characteristics}

A sensor node usually consists of four sub-systems. The Sensing the Computing, the Communication, the and the Power supply subsystem. All these systems participate in order to fulfill the tasks of a single Sensor which will finally offer to the Network its data for processing [10]. Also, the number of SRs which form a Network might vary. Another issue which have to be to tackled is the number of sensors nodes which are required in a given area.That is, determining the network density. In [11] network density is defined as :

$$
\mu(\mathrm{R})=\left(\mathrm{N} \cdot \pi \cdot \mathrm{R}^{2}\right) / \mathrm{A}
$$

where, $\mathrm{N}$ is the number of scattered nodes in a region $\mathrm{A}$, and $\mathrm{R}$ is the radio transmission range, $\mu(R)$ gives the number of nodes within the transmission radious of each node in region A. Addition of extra nodes does not provide additional sensing nor coverage fidelity, beyond a critical value $\lambda$. Hence techniques would be required to decide optimized deployment for an area of interesting.

\subsubsection{Sensor states in WSNs}

During cooperation between sensors and the base station of the network in WSNs the SRs can be in four different states as follows:

1) Sensing: SR is monitoring, digitizing, processes the information and then stores the data which will be eventually sent back to the base station.

2) Relaying: The SR receives data from other SRs and forwards them towards the base station.

3) Sleeping: A sleeping node does not participate in either sensing or relaying.Most of the device is either shut down or works in a lowpower mode. However, from time to time it might change its state and "wake up" in order to listen to the communication channel and answer requests from other nodes. Depending upon the type of the received request it's state transition to "sensing" or "relaying".

4) Dead: A dead node has either used up its energy or has suffered a vital damage. Once a node is dead, it is no longer available to the sensor network and it cannot re-enter any other state [12].

2.2.2 Recovery from SRs failure in a Wireless Sensor NetworkWSN
A SR failure or the simultaneous failure of a number of SRs in a WSN might create disjoint segments and the missing of critical information. There exists a significant amount of work related with connectivity recovery. In most of work done already a group of SRs or different groups contribute in the recovery process by their movement.

In a WSN where SRs are movable [13] had presented a novel distributed algorithm, Autonomously Repair AuR which enables a network to restore connectivity by only local coordination among SRs in the individual segments. AuR models electrostatic forces of attraction and repulsion. The neighbors of a failed node to collaboratively decide on how to bridge the gap that a loss introduces by moving one or multiple healthy nodes. Self-spreading of nodes and movement toward the center of the deployment area offers recovering from a multi-node failure.

\section{Localization in WSNs}

The two main categories of localization in WSNs are the Target/source localization and the Node self localization.

\subsection{Target/source localization}

Target/source localization can be classified into two main categories depending on the area of interest. These two categories are the indoor and outdoor environment. In the indoor environment the target might be a human or a device which is moved in a house whilst in the outdoor the target might be a vehicle or an aircraft. There are also cases of underwater localization. In this case the target might vary depending upon the type of animal under research. The target might be a sea animal like a seal or a whale which are under research.

\subsection{Single-Target/Source Localization in Wireless Sensor Network.}

There are several ways to estimate the source location: Angle of arrival (AOA) [2],

Time Difference of Arrival (TDOA) [3-6],

Energy-based. Energy-based method is an attractive method because it requires low hardware configuration and that makes it an inexpensive approach.

Single-source localization can be further divided into: energy decay model-based localization algorithm and model independent localization algorithms.

\section{(1) Decay Model-Based Localization Algorithm. Equation}

Although this energy decay model appears quite simplistic, it is the one commonly used in the literature.

Since the objective function of single-source localization method has multiple local optima and saddle points [7], the authors formulated the problem as a convex feasibility problem and proposed a distributed version of the projection onto convex sets method.

A weighted nonlinear least squares and weighted linear least squares methods [8] were proposed to estimate the location of the target. In [9], the authors proposed normalized incremental subgradient algorithm to solve the energy-based sensor network source localization problem where the decay factor of the energy decay method is unknown. Unlike the signal models in [7-9], the authors derived a more generalized statistical model for energy observation 
[14], and a weighted direct/one-step least-squares-based algorithm was investigated to reduce the computational complexity. And in comparison with quadratic elimination method, these methods were amenable to a correction technique which incorporates the dependence of unknown parameters leading to further performance gains. This method offered a good balance between the localization performance and computational complexity. Energy ratio formulation [11] was an alternative approach that is independent of the source energy $S(t)$. This was accomplished by taking ratios of the energy reading of a pair of sensors in the noise-free case. In [12], the authors proposed an energy aware source localization method to reduce the energy consumption in localization.

\section{(2) Model-Independent Methods.}

In [16] cited by Cheng, et al.[17] a novel model-independent localization method was proposed. Since the nodes with higher received signal strength measurement were closer to the source, a distributed sorting algorithm is employed. If the sensor nodes know their rank, the required distance estimates are obtained as the expected value of the respective probability density functions. Finally, the projection onto convex sets (POCS) method was used to estimate the location of the source.

\subsection{Multiple-Target Localization in Wireless Sensor Network.}

Many works investigate the single-target localization. However, very limited papers investigate the multiple-target localization. Most of the works are based on the maximum likelihood estimator. And a multi resolution (MR) search and the expectation maximization (EM) method were also proposed [17].

\subsection{Range-Based Localization.}

The classic methods to estimate the indoor location are time of arrival (TOA), Time Difference of Arrival (TDOA), Angle of Arrival (AOA), and Received Signal Strength (RSS). TOA method measures travel times of signals between nodes. TDOA method locates by measuring the signals arrival time difference between anchor nodes and unknown node. It is able to achieve high ranging accuracy. The disadvantage of this method is that it requires extra hardware and consumes more energy. We could mention that for a large network the energy consumption is high and this is an issue that have to be tackled in advance.RSS has established the mathematical model on the basis of path loss attenuation with distance. It requires relatively low configuration and energy and it is an inexpensive approach. AOA, is a method in which the angles between unknown node and a number of anchor nodes are used to estimate the location. This method needs the antenna array which is an expensive solution for low-cost sensor node.

\subsection{Range-Free Localization}

Range free techniques prove to be the best option in WSNs networks as only a fraction of sensor nodes (called anchor nodes or beacon nodes) are employed with GPS.SRs that doesn't know their location, (unknown nodes) calculate their position using anchor nodes by a process called node self localization or cooperative localization. The high cost of hardware for range based approaches in WSNs lead to range-free localization solutions as a cost-effective alternative. In this case absolute point-to-point distance estimates are of second importance. In [19], four Range-Free algorithms are presented (Centroid Algorithm, CPE Algorithm,APIT Algorithm , DV-hop algorithm) with comparison of various attributes Fig.1 showed that
DV Hop algorithm is efficient in larger sensor network with greatest accuracy whilst the Centroid algorithm is very cost effective [18],[21].

\begin{tabular}{|c|c|c|c|c|c|}
\hline S No & Type of localization algorithm & Accuracy & GPS Error & $\begin{array}{c}\text { Number of Neighbor } \\
\text { Anchors }\end{array}$ & Overhead \\
\hline 1 & $\begin{array}{c}\text { Centroid } \\
\text { Algorithm }\end{array}$ & Fair & Good & $\geq 3$ & Smallest \\
\hline 2 & CPE algorithm & Fair & Fair & $\geq 3$ & Small \\
\hline 3 & $\begin{array}{c}\text { APIT } \\
\text { Algorithm }\end{array}$ & Good & Good & $\geq 3$ & Large \\
\hline 4 & DV-hop algorithm & Good & Good & No restriction & Largest \\
\hline
\end{tabular}

Fig. 1 Range-Free algorithms comparison

\subsubsection{Hop-Count-Based Localization.}

DV-Hop is the typical range-free positioning system. It does not need to measure the absolute distance between the beacon node and unknown node. It uses the average hop distance to approximate the actual distances and by that way it reduces the hardware requirements.

Its implementation is easy and can be applied to a large network. The problem is that the positioning error is correspondingly increased. The positioning process of DV-Hop is divided into three stages:

\section{-Information broadcast}

\section{-Distance calculation}

\section{-Position estimation}

In information broadcast stage, the beacon nodes broadcast their location information package which includes hop count and is initialized to zero for their neighbors. The receiver records the minimal hop of each beacon nodes and ignores the larger hop for the same beacon nodes. Then the receiver increases the hop count by 1 and transmits it to neighbor nodes. All the nodes in a network can record the minimal hop counts of each beacon nodes. In distance calculation stage, according to the position of the beacon node and hop count, each beacon node uses the following equation to estimate the actual distance of every hop: where $(x i, y i)$ and $(x j, y j)$ are the coordinates of beacon nodes $i$ and $j$, respectively. $h j$ is the hop count between the beacon nodes.

Then, beacon nodes will calculate the average distance and broadcast the information to network. The unknown nodes only record the first average distance and then transmit it to neighbor nodes. In position estimation stage the unknown node calculates its location.

\subsubsection{Pattern Matching Localization}

One of the most viable solutions for Range-Free localization methods recently is the Pattern matching localization, also called Fingerprint or Map-based algorithm. The fingerprint localization has two phases. In the first phase, the received signals at selected locations are recorded in an offline database called radio map with RSSI In the second phase, it works at the online state and match online measurements with the closest a priori location fingerprints. But when using the fingerprint-based method, a commonly used method of estimating user location is to find the nearest reference point, using the Euclidean distance in signal space. Euclidean distance method is prone to error, especially when Wi-fi access points are unstable: a problem that frequently might occur. Another issue is that some access points may be active when generating radio map and not 
active when their location is estimated. In addition, even if an access point is active, the user may not receive signal due to problems such as collisions. When the signal strengths from unstable access points are fed into the standard estimation algorithm, they can lead to serious estimation errors.Also, an observation is made that RSSIs from access points can be shifted due to various reasons height difference with the user [17],[20].

\subsubsection{Range Free -RF Localization Algorithms}

During the last decade a number of algorithms have been proposed in order to tackle the localization problem with a RF method in WSNs.

In the Point-In-Triangulation Test (PIT) Fig.2, a node chooses three anchors from all audible anchors (anchors from which a beacon was received) and tests whether it is inside the triangle formed by connecting these three anchors. This test is used as a basis for many algorithms to find the possible area in which a target node resides.
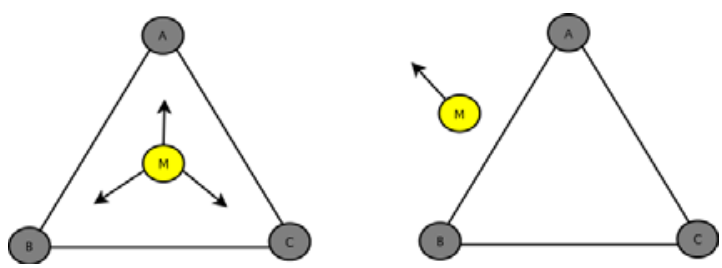

Fig. 2 PIT location Test

He et al. proposed APIT [21], where an unknown node position is estimated as the center of gravity of the triangles overlapping region Fig. 3. The triangles are formed, by connecting all three anchor nodes heard by unknown node,and PIT test is applied for each triangle. This algorithm requires a low hardware, however, the accuracy of localization in this method is affected by a node's presence whether it is within the triangular regions or not [22] .

The Approximate Point in Triangulation APIT is one of the typical PIT algorithms based-on regional determination. However, APIT algorithm requires anchors in a single hop, high nodes' density and anchors' uniform distribution. Yet nodes' density is seldom high and nodes' distribution is uneven in actual WSN networks, in which the accuracy of APIT only reaches to about $35 \%$.Thus, APIT algorithm requires harsh conditions and its accuracy is not high.

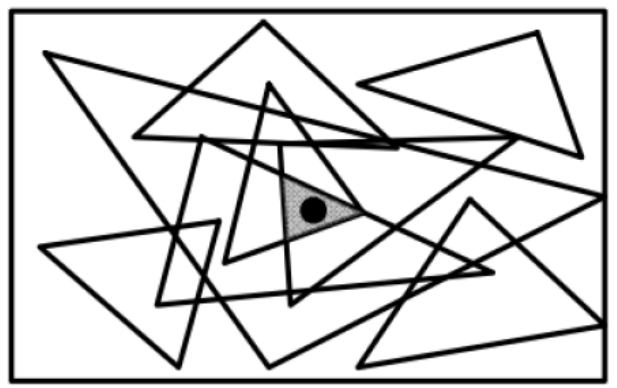

Fig. 3 APIT location Algorithm

Xu et al. ,[23] introduced a Novel PIT Localization Algorithm based on Coverage of Anchors. The novel algorithm improves the accuracy of about $20 \%$. by determining whether the test point is inside the triangle formed by three anchor nodes through judging whether the center of gravity of the intersection of the anchors' coverage area is inside the triangle Fig 4.

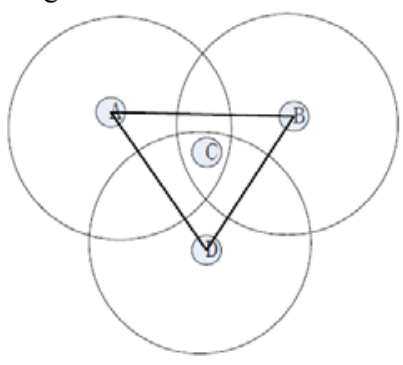

Fig. 4 Schematic diagram of the Novel PIT Algorithm

Wu et al., [24] combined the RSSI (Received Signal Strength Indication) and APIT (Approximate Point in Triangulation) algorithm to improve APIT algorithm. They showed that APIT has some errors in the localization procedure and proposed the Improved APIT algorithm, IAPIT. To solve the problem of location precision that exists in the APIT location algorithm, RSSI was introduced to revise the location precision. For the anchor nodes within one unit of the unknown node, they used RSSI to measure the distances between the unknown nodes and the anchor nodes and then selected three anchor nodes with smaller distances, and calculated the estimated position by using trilateration measurement. Then they calculated the center of mass of the overlapping region as the estimated position, according to APIT algorithm. Finally they calculated the average value of these two positions as the final position coordinates. For the unknown nodes that were unable to be located by APIT algorithm, they used RSSI ranging quantitative model to assist location and find the estimated coordinates of the unknown node. As the APIT algorithm suffers from significant position errors due to theoretical deffects and and RSSI inaccuracy, Xiaofeng Li et al. [25] presented the FAPIT, which is a combination of IAPIT and a Fuzzy logic system, where the SRs coordinates are estimated by using the Centers of Gravity COGs of the triangles intersection areas. By presenting the defects of the APIT and the IAPIT the proved that the FIAPIT works better as the fuzzy logic inference based on IAPIT helps to manage relative uncertainties which are associated with irregular RSSI. They also showed that IAPIT works better than APIT when the Node Density is large enough. High level of FAPIT is depicted in the following Fig.

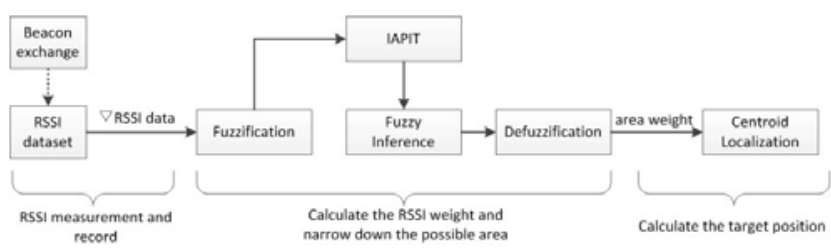

Fig. 5 High level of FAPIT

X.Sun et al. [26], tried to improve the accuracy of the node localization in WSNs and proposed a new range-free localization using Voronoi diagrams based on the Approximate Point-InTriangulation test (APIT) algorithm. Their design of Voronoi PointIn-Triangulation VPIT mainly consists of P.I.T test and Voronoi diagrams, then is following the stage of calculation of the overlapping region center of gravity. The work-flow diagram of 
VPIT is depicted in Fig. 6.

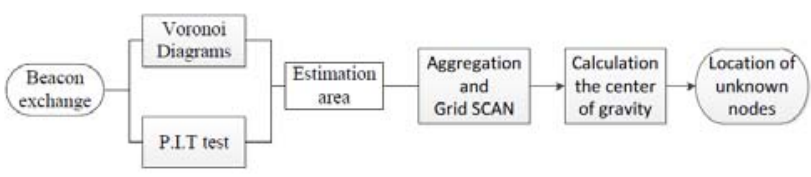

Fig. 6 VPIT Work -flow diagram

They compared their algorithm with APIT. Their simulation results showed that their VPIT algorithm improved the precision of localization by narrowing the node's geometry region.Compared with APIT, the estimation error of VPIT algorithm was lesser and with better performance.

\section{Localization in Telemetry}

Another type of localization is the localization in telemetry where researchers use bearings to locate species of animals. Telemetry for the observation and research of wildlife was introduced more than 50 years ago, by Adams 1965, and Cochran et al., 1965 [27], [28]. It is applied localization through triangulation by radio tracking in areas of surveillance, mostly where species of animals behavior and movements are under investigation. One of the common known method is the "ad hoc" method, in which the location is estimated to be somewhere in the middle of the intersections of several bearings. The ad hoc method is easy to use, but neither the location nor the error estimates are objective.

Another technique is the "error polygon method" which was introduced by Heezen et al. cited by Nams et al in which an error polygon is represented as the intersection of the arcs formed by the confidence intervals of 2 bearings. This method is restrictive in that it only measures precision of individual locations for 2 bearings [29].

Heezen et al. in their research of three deer movements [30], showed that as the distance from the base line of detection increases there is an increase in the size of the error polygon, $\mathrm{d} 1>\mathrm{d} 2$ Fig.7.

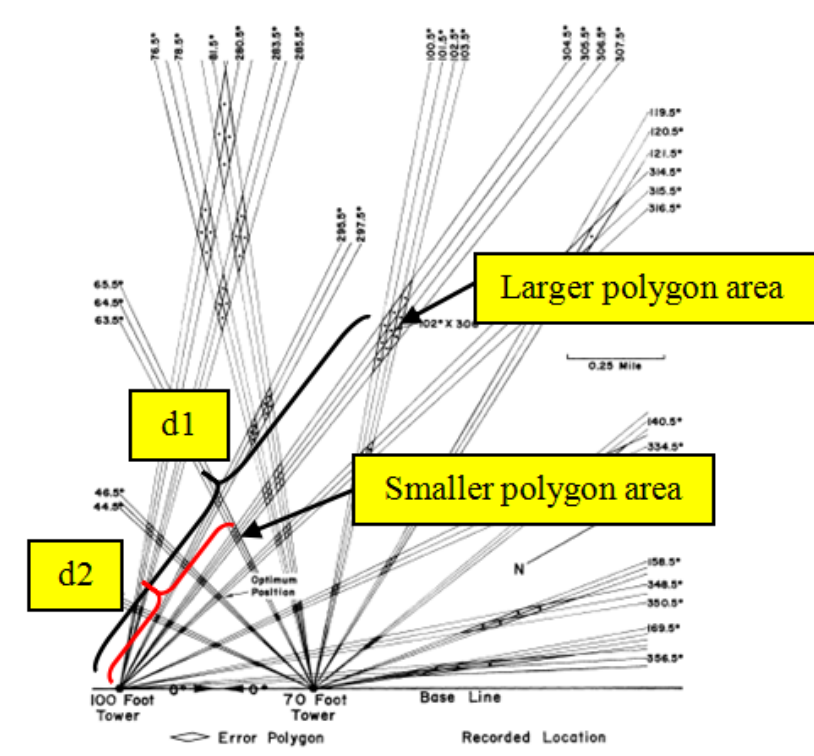

\section{Network Topology}

Every Sensors network has a different topology depending on its type and purpose. Recent years there is an extensive ongoing research related with network topologies which might offer a great amount of coverage, energy efficiency and internal network communication.

Fig. 7 Relative sizes and shapes of error polygons at selected locations in relation to the two tracking towers. Any true location within an error polygon would be recorded at the center of that polygon.

The third method, which is "the error triangle technique" uses 3 bearings and defines the error polygon by the points at which the bearings from the receiving antennas cross rather than the intersection of the error arcs. The problem with this technique is that although one can measure the area of the triangle, there is no suggested way to estimate the probability of the true location being within the Triangle, and there is no probability value given to the size of error triangles.

The fourth method developed by Lenth cited by Nams et al. uses maximum likelihood estimators to estimate both location and precision. The Lenth maximum likelihood estimators objectively estimate both location and precision of the location for any number of bearings.

In relevance with the telemetry error in localization, Montgomery et all, [31] pointed out that telemetry error, patch size and categorical raster resolution interact with each other and influence the accuracy of assigning co-variate values to estimated wildlife telemetry locations. They quantified the analogy of Telemetry errors as it is described below:

-The probability of being correct for all patch sizes, will generally have $>50 \%$ for telemetry errors up to $60 \mathrm{~m}$.

-Accuracies $>90 \%$ tend to be restricted to intermediate telemetry errors $(10 \mathrm{~m}<$ telemetry error $<36 \mathrm{~m})$ at larger patch sizes $(20-200$ ha) or small telemetry errors (telemetry error $<10 \mathrm{~m}$ ).

They also provided metrics and a replicable methodology by which researchers and managers are able to identify the relative accuracy of wildlife models given the mean error of their telemetry system and the patch size characteristics of their study. Ward et al. [32] in their research with an automated telemetry system and by using arrays of single Automated Receiving Units (ARUs) they were detecting positions of snakes and their movements. A snake's position by using an ARU array was determined with bearings from each of the ARUs and estimation of the location based on the intersection of those bearings. The spatial accuracy of the system was affected by a number of factors, like the strength of the transmitter,height of the transmitter above the ground, its orientation to the ARU antennas, the density of vegetation or other structures between the transmitter and the ARU. They concluded that the most obvious advantage of tracking snakes using ARUs was that automation greatly increased the amount of data that could be collected per individual and potentially the number of individuals that can be tracked simultaneously, with much less human involvement. Also, and as data were collected around the clock and potentially for as long as transmitters were operating, the activity and movement profiles generated were far more complete than would be possible with conventional tracking, meaning the hand tracking.

Some shortcomings of using the ARUs were that the targets (snakes) had to remain within their detection range, and unlike hand tracking, where a snake's position could be determined within a few meters locations determined by ARUs had errors of tens of meters at best. They commented that due to limitations of that technology and in order to become most effective it should be coupled with both appropriate species and appropriate questions and future research seems likely to benefit from using a combination of the two technologies (Conventional and automated). 
Topology should be carefully designed in order for the base station to receive and not miss the sensors data. As it is depicted in Fig. 8 there are a lot of topology issues that should be examined carefully when a WSN will be designed.

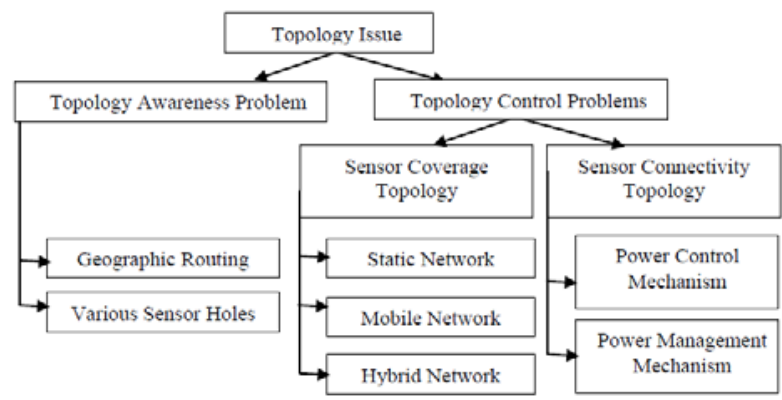

Fig. 8 Taxonomy of topology issues in WSNs

\subsection{Wireless sensor networks topologies}

In the Wireless sensor networks the topologies are the Bus, Tree, Star, Ring, Mesh, Star-Mesh ,Circular and Grid. Each one of them has its own performance and contributes in the energy conservation of the system, whilst the Grid topology appears to be the most energy efficient [33]. Nivedita et al. [34] present different basic network topologies and as every topology has its own advantages and disadvantages they suggest that a combined topology known as Hybrid topology can be used which is reliable, scalable, flexible and effective. The only disadvantage is the complexity of its design and a costly infrastructure which results from the combination of two or more different topologies.

\subsubsection{Mesh Topology}

Wireless mesh networks Fig. 9 were originally developed for military applications. Mesh networks are typically wireless. As over the past decade, the size, cost, and power requirements of radios has declined, enabling multiple radios to be contained within a single mesh node, thus allowing for greater modularity. Each node can handle multiple frequency bands and support a variety of functions as needed [35].

One of the main advantages of this topology is that there is no single point of failure. The chances of data loss are very less as an alternate path can be found in this type of topology for the nodes. That makes it the most reliable communication network structure whilst synchronously it is a scalable network. The disadvantage with this type of network is that there are too many redundant paths and that for a wireless sensor network all the point-to-point links must be tuned. Overall this results in high power consumption.

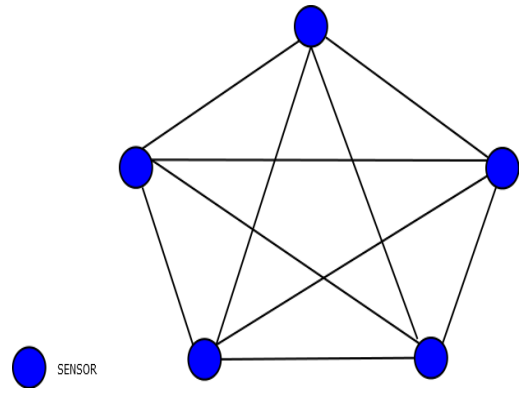

Fig. 9 - Mesh Topology

\subsubsection{Tree Topology -}

All the sensors construct a logical tree and for that reason we call this topology a Tree Topology Fig 10. In this topology we have two types of nodes; one is a parent node and the other is a leaf node. Data packets are passed from a leaf node to its parent nodes. The advantage of this topology is that it consumes less power than other topologies, whilst one a of its main advantages is that if a node fails then the entire sub-tree is cut-off from the base station. Adding to that that it is time consuming and costly as the data have to be transmitted from the leaf nodes to the root node we would say that this topology doesn't looks promising and trustful.

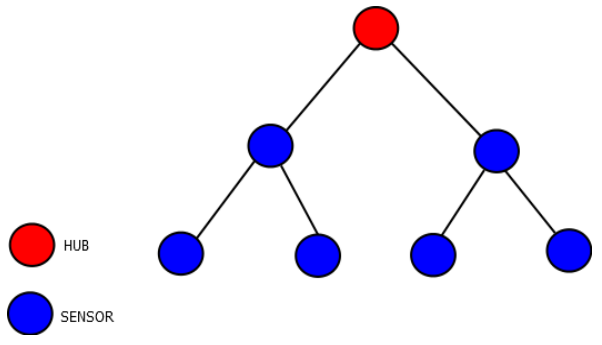

Fig. 10 - Tree Topology

\subsubsection{Star Topology}

In this topology Fig.11, compared with the Mesh topology we have lower power consumption and this topology is easy to enlarge which makes this topology structure scalable. The main disadvantages of this topology for a wireless sensor Network are that there is no reliable communication due to a single point failure and there is no alternate path in this structure of any node [36].

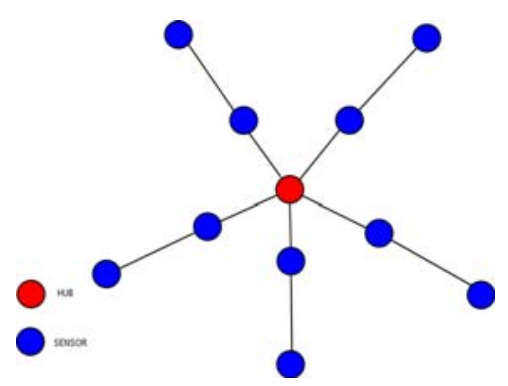

Fig. 11- Star Topology

\subsubsection{Star -Mesh Topology}

Star-Mesh topology, Fig. 12 is an hybrid topology that combines the advantages of star topology and mesh topology. Among all nodes, one node is specified as the PAN coordinator and with it the network can be identified. This hybrid topology organizes the nodes in star topology around mesh nodes, which they finally turn into a 
mesh network. The hybridization of Star-Mesh network offers the highest degree of sensor node mobility and flexibility for fast changes in the network population and the overall low power consumption. For this reason, the star - mesh hybrid has proved to be logical choice for many implementation of wireless sensor networks [37].

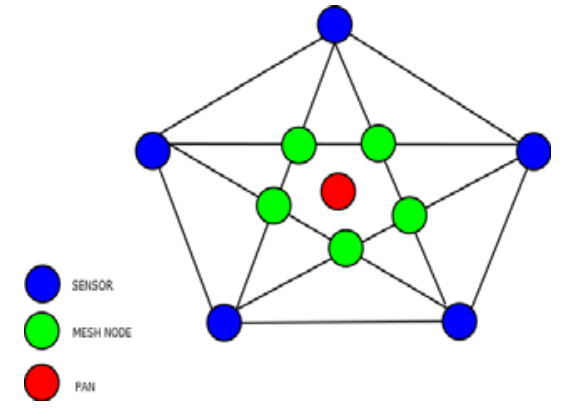

Fig. 12 - Star -Mesh Topology

\subsubsection{Ring Topology}

In this topology Fig. 13 only neighboring nodes are able to communicate with each other. One advantage of this sensor network topology is that it does not have any leader (central node), but if any node or link is broken during the communication, then it will affect the whole sensor network. The main disadvantage is that data packets must pass through every node between the sender and recipient and that makes the network slower. Additionally, if any of nodes fail then the ring is broken and data cannot be transmitted successfully. For that reason nowadays, ring topology is not preferred much in applications.

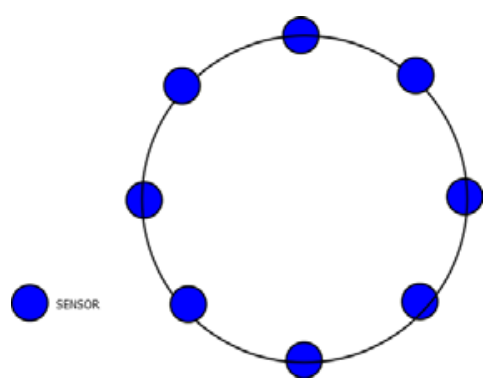

Fig.13 - Ring Topology

\subsubsection{Circular Topology}

In this topology, Fig.14 there is a sensing area which has a sink at the center. SR nodes sense the area and when they sense an event they transmit data to the sink. Nodes are randomly deployed with uniform density all around the sink as shown in Fig. 12 . Circular web topology is more efficient,easy to establish and easy to maintain [38], [39].

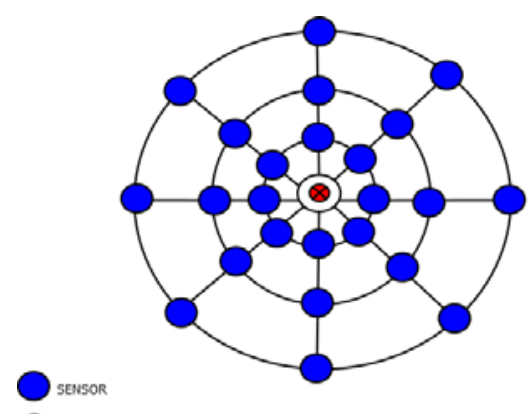

Fig. 14 - Circular Topology

\subsubsection{Grid Topology}

In this topology the sensor network field is divided into grids as it is depicted in Fig.15. The network area is partitioned into nonoverlapping square grids of the same size. In each grid there should be at least one and only one node in working state at any time. The nodes in a grid should work in turn in order to extend the network life time. In each grid, there is one node which is selected as a grid head and which is responsible for forwarding routing information and transmitting data packets. Routing is performed in a grid-by- grid manner. A Grid-based multi-path routing protocol intended to route packets fast, utilize and extend sensor nodes energy in addition to avoiding and handling network congestion when happens in the network [38].

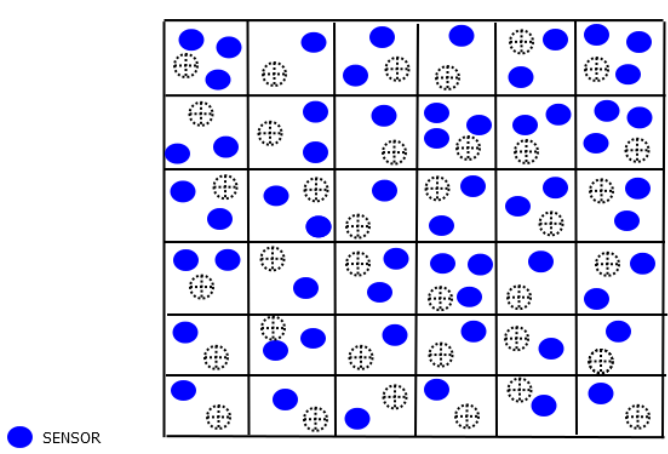

SENSOR

Fig. 15 - Grid Topology

\subsubsection{Bus Topology}

Bus topology Fig 16, is easy to install and in this topology, each node sends a message to another node on the network. In this topology a node broadcasts message if it wants to send any information to any other node in network. All nodes see or receive the message but only recipient actually processes the message and the rest nodes discard the message. As it has a single path communication traffic congestion may easily make the network unavailable. Bus networks work best with a limited number of nodes. If more than a few dozen nodes are added to a network bus, performance problems will likely result.It cannot be counted as scalable as it has a limited length and range and is relatively slower [38],[40]. 


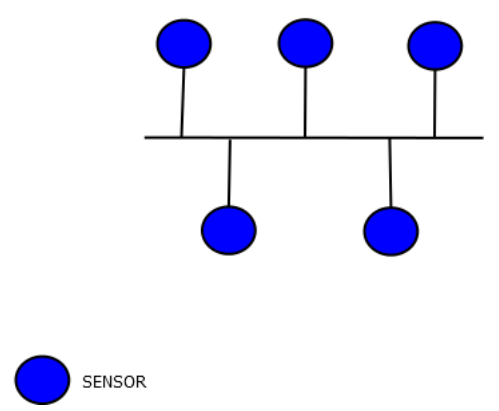

Fig. 16 - Bus Topology

\subsection{Topology Control}

Topology Control -TC allows the maintaining of the communication links between network nodes and their connectivity. Also, it characterizes the group of network nodes, adding or deleting nodes in the group. Transmission range and scheduling of nodes alter the topology of sensor network. By controlling the topology more energy can be saved for the network. For that reason TC plays an important role in energy conservation and might increase the life time of overall network.. But a network in order to achieve its purpose and remain reliable it has to deal with a various TC problems. The TC problems can be divided into two categories: Sensor Coverage Topology and Sensor Connectivity Topology [13].

\subsubsection{Sensor Coverage Topology}

The coverage topology describes the topology of sensor coverage and is concerned about how to maximize a reliable sensing area while consuming less power. The SRs locations can be predefined in the sensor field or might be positioned at places which are more weighted and are critical for monitoring [41]. There are three categories into Sensor Coverage Topology: Static , Mobile and Hybrid .

\subsubsection{K-coverage}

In many applications where it is needed a strong coverage capability every point in the area (monitored or tracked by sensors) should be covered by at least a fixed parameter of $\mathrm{k}$ sensors, where $\mathrm{k}$ is a predefined value. In a case that $\mathrm{k}=3$ every point should be covered by a minimum of three SRs or more.Huang and Tseng (2005), proposed a novel solution to determine whether a sensor network is $k$-covered. They checked if the sensing range of each sensor can be a unit disk $k$-Unit-disk Coverage $(k-U C)$ or a $k$-Nonunit-disk Coverage ( $k$-NC) Problem. Their approach looked at how the perimeter of each sensor's sensing range is covered or not, and proved that the whole area is sufficiently covered as long as the perimeters of sensors are sufficiently covered. The $\mathrm{k}$ coverage problem have been extensively studied the last years [42] - [45], and specifically for the positioning with triangulation problem triangulation-based positioning protocols [45] - [47] require at least three sensors (i.e., $k \geq 3$ ) to monitor a moving object in the sensing area of interest [48], [49].

\subsubsection{Static Coverage}

The Partial Coverage where only a necessary set of sensors is working and the second is the Single Coverage. A static network must be deployed according to a predefined shape. The predefined locations of the sensors can be uniform in different areas of the sensor field or can be weighted to compensate for the more critically monitored areas [44].

\subsubsection{Mobile Coverage}

Different from static coverage, the nodes here use dynamic coverage and a group of nodes or all of them have the ability of moving. In this case, when an area is given to be monitored, the proposed distributed self-deployment protocols first discover the existence of coverage holes in the target area then calculate the target positions. Then they move sensors in order to diminish the coverage holes.

\subsubsection{Hybrid Coverage}

In this type of Coverage, there exist SRs that are capable for movement.Those SRs can help in deployment and network repair by moving to appropriate locations within the field to achieve desired level of coverage. It is a combined solution for the exploration and coverage of a given target area. A coverage problem can be solved with the help of a constantly moving SR or a set of SRs in a given target area of interest. The available mobile sensors in a hybrid network might be moved to heal coverage holes. All decisions are made by the moving SR by directly communicating with a neighboring SR.

\subsubsection{Sensor Connectivity Topology}

The connectivity topology on the other hand is more concern more about network connectivity and emphasizes the message retrieve and delivery in the network. Two kinds of mechanisms have been utilized to maintain an efficient sensor connectivity topology:

\section{Power Control Mechanisms}

Parameters which are used to evaluate the TC of a network are: Connectivity, Energy efficiency, Throughput and the Network lifetime [50].

\section{Connectivity}

The multiple path between nodes should be maintained and the connectivity between nodes to share messages should be on the right percentage which will allow all the messages to reach the base station.

\section{Energy efficiency}

The two factors that determines the energy efficiency of the network are the "Energy stretch factor" and the "Hop Stretch factor. Also, the distance between nodes influences proportionally the energy consumption for transmission and reception between nodes.

\section{Network lifetime}

The overall networks lifetime is required to remain high in order to maintain the connectivity and coverage of the network. Nodes failure to balance energy consumption might decrease the network lifetime or even worse lead to a network failure.

\subsection{Topology awareness}

Topology Awareness is another issue of topology and includes the geographic routing problem and the sensor holes problem. Failure 
use adequate geographical and topological information will result in low routing efficiency and high power consumption. This eventually, might easily lead to network degradation and to its inefficiency.

\subsubsection{Geographic Routing}

Geographic routing uses geographic and topological information of the network to achieve optimal routing schemes with high routing efficiency and low power consumption. SR holes, such as Jamming holes, sink/black holes and worm holes, may form in a WSN and create network topology variations which trouble the upper layer applications. In a network with a great number of nodes in which intense communication is taking place this may result in the creation of jamming holes and failure message delivery to the exterior nodes. Another type of holes are the Sink/Black holes and Worm holes which are caused by nodes exhausted around sink node or pretended sinks or by malicious nodes. SR holes issues should be treated carefully, as they will create a costly routing table and will exhaust the intermediate nodes rapidly.

\subsubsection{Network Holes}

Whenever in an area of the network either nodes are not available or the available nodes cannot participate in the actual routing of the data due to various reasons, a routing hole is created. Sink/Black holes and Worm holes are related with the power consumption in the network and are gradually formed due to sensor node power exhaustion and possible denial of service attacks in the network. Prevention of data loss and reliability of the network is ensured with Hole detection and

The various types of holes are the following:

\subsubsection{Coverage holes}

Coverage holes are formed due to bad design or unsystematically area arrangement.Also,poor installment, weak power of nodes or topology failure due to the presence of obstacles, might lead to the appearance of a coverage hole, [51]. In [52], there was discussed and analyzed a system, Fig 17, to deal with the holes problem and ways to heal the network.Hole healing is done by moving the nearby nodes with high energy. Neighboring nodes are selected in such a way that network connectivity is not disturbed. During the healing process, the originator finds the nodes with minimum distance and moves them to a particular distance.And by this process the hole is healed.

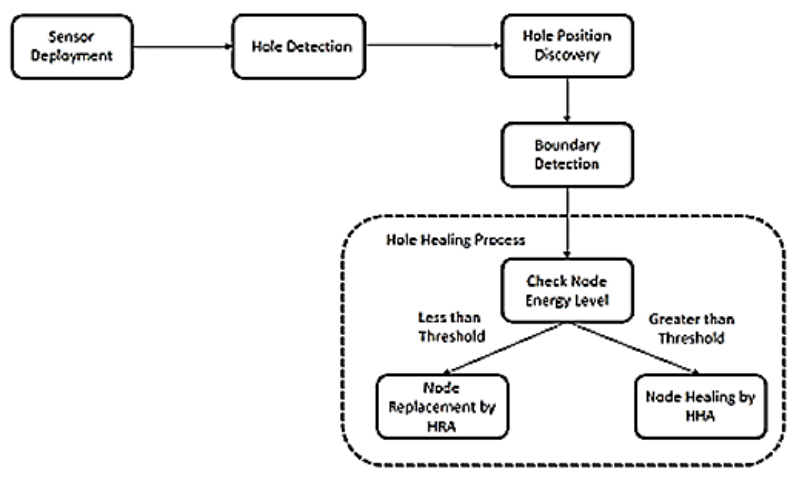

Fig. 17

\subsubsection{Jamming holes}

Jamming holes are the type of holes which circumvents the ability of nodes in a specific area to communicate and sense and by that way a virtual hole emerges. Jamming can be deliberate or unintentional. The zone of influence centered at a jammer is referred as a jamming hole. Unintentional jamming results when one or more of the deployed nodes malfunction and continuously transmits and occupies the wireless channel denying the facility to other neighboring nodes. Though in deliberate jamming an adversary is trying to impair the functionality of the sensor network by interfering with the communication ability of the sensor nodes [53].

\subsubsection{Sink/Black holes}

Sinkhole/Black holes is a type of attack were compromised node tries to attract network traffic by advertising a fake routing update. The attacker listens to request for routes and then replies that it contains the shortest path to the base station. Once the malicious device inserts itself between the sink and the sensor node it can do anything with the data packets passing between them, Fig 18 . One of the impacts of sinkhole attack is that, it can be used to launch other attacks like selective forwarding, acknowledge spoofing and drops or altered routing information. It can also used to send bogus information to base station [54].

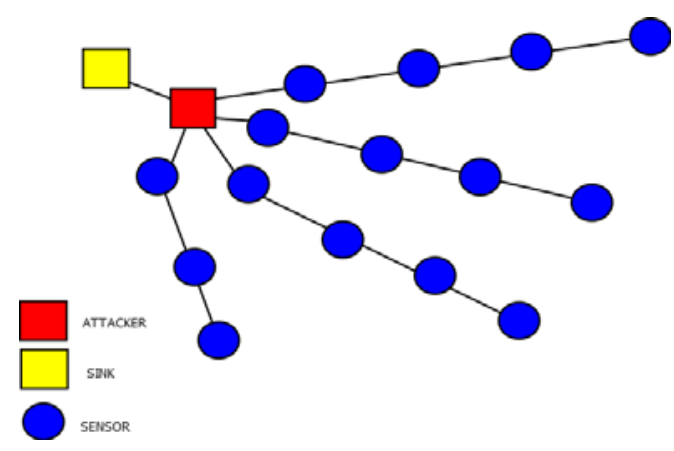

Fig. 18

N. Gandhewar et al. in [55], suggests a mechanism to identify sinkhole attacks by checking for very large variations in the sequence numbers for the Request packet. They discussed the AODV protocol and they showed its performance with no sinkhole attack, under attack \& after applying a mechanism.

\subsubsection{Worm holes}

Worm holes are formed when a malicious node causes nodes located in different parts of networks to believe that they are neighbors. This results in incorrect routing convergence and communication overhead [56]. Wang et al. in [57] proposed MDS-VOW, a mechanism, to detect wormholes in a sensor network. MDS-VOW does not require the sensors to be equipped with special hardware, and adopts and combines techniques from social science, computer graphics, and scientific visualization to attack the problem in network security. Results showed that MDS-VOW has a low false alarm ratio when the distance measurement errors are not large. 


\section{Conclusion}

As a conclusion we can mention that for a fixed stations network which has to perform in an autonomous way a lot of parameters should be examined carefully. This research shows that not only sensor types, or their topology affect a network performance but also careful consideration should be given to the network purpose. If it is a network for monitoring or a network for military surveillance its parameters might differentiate in order to reach a high rate of appropriateness. Also, hole types might decrease its performance this is a network issue that ought to be examined carefully. Furthermore, a certain pattern of arrangement of nodes is also related with the efficiency of a $\mathrm{SN}$, and a proper arrangement is required. A better arrangement results in a more efficient network. Additionally, a more accurate system able to calculate the data of many SRs is needed, which will also enable researchers to process triangulations of SRs that use technology of telemetry for localization. Future work might involve other network attributes like scalability and SRs power.

\section{REFERENCES}

[1] M. Sfendourakis N. Rajaponal E. Antonidakis "Automatic Triangulation Positioning System for wide area coverage from a Network of Stations in Fixed Positions".In Proceedings of the Zakynthos ICCS , Jul 2015.

[2] Gast, M.S. (2002). 802.11 Wireless Networks: The definitive Guide. 1st ed. O’Reilly.

[3] S. Sharmal, D. Kumar and K. Kishore "Wireless Sensor Networks- A Review on Topologies and Node Architecture" - JSCE Vol.-1(2), pp (19-25) Oct 2013.

[4] Rajiv Kr. Tripathi, Yatindra Nath Singh and Nishchal Kr. Verma, "Two tiered wireless sensor networks - Base station optimal positioning case study," Published in IET Journal Wireless Sensor System, Vol.2 Issue 4 December 2012, pp. $351-360$.

[5] https://en.wikipedia.org/wiki/Wireless ad hoc network accessed March 272016

[6] A. Howard, M. J. Mataric and G. S. Sukhatme, "Mobile Sensor Network Deployment using Potential Fields: A Distributed, Scalable Solution to the Area Coverage Problem," 2002.

[7] P. Flocchini, G. Prencipeb, Nicola Santoroc Self-deployment of mobile sensors on a ring" - Theoretical Computer Science Volume 402, Issue 1, 28 July 2008, Pages 67-80.

[8] J. Teng, T. Bolbrock, G. Cao and T. la Porta, "Sensor Relocation with Mobile Sensors: Design, Implementation, and Evaluation," Mobile Adhoc and Sensor Systems, 2007. MASS 2007. IEEE International Conference on, Pisa, 2007, pp.

[9] G.Wang, G. Cao, and Tom LaPorta"Movement - assisted sensor deployment”. In IEEE INFOCOM ,June 2004.

[10] V. Raghunathan, C. Schurgers, Park.S, and M.B. Srivastava, "Energy-aware wireless microsensor networks," IEEE Signal Processing Magazine, Volume: 19 Issue: 2 , March 2002 Page(s): $40-50$.
[11] N. Bulusu, D. Estrin, L. Girod and J. Heidemann, "Scalable Coordination for wireless sensor networks: Self-Configuring Localization Systems," In Proceedings of the Sixth International Symposium on Communication Theory and Applications (ISCTA 2001), Ambleside, Lake District,UK, July 2001.

[12] W. Li and C. G. Cassandras, "A Minimum-Power Wire- less Sensor Network Self-Deployment Scheme," IEEE Wireless Communications and Networking Conference, Vol. 3, 2005, pp. 1897-1902.

[13] Yatish K. Joshi and Mohamed Younis Autonomous Recovery from Multi-node Failure in Wireless Sensor Network Globecom 2012- Ad Hoc and Sensor Networking Symposium

[14] Nadeem Ahmed Salil S. Kanhere1 Sanjay Jha "The Holes Problem in Wireless Sensor Networks: A Survey" Mobile Computing and Communications Review, Vol 9, Number 2

[15] P. Basu and J. Redi, "Movement Control Algorithms for Realization of Fault-Tolerant Ad Hoc Robot Networks," IEEE Networks, 18(4), pp. 36- 44, Aug.2004.

[16] Younis Mohamed, Akkaya Kemal "Strategies and techniques for node placement in wireless sensor networks: A survey Original Research Article" $A d$ Hoc Networks, Volume 6, Issue 4, June 2008, Pages 621-655

[17] Cheng, L., Wu, C., Zhang, Y., Wu, H., Li, M. and Maple, C., 2012. A survey of localization in wireless sensor network. International Journal of Distributed Sensor Networks, 2012.

[18] Kaur, R. and Malhotra, J., 2015. Range Free Localization Techniques for Randomly Deployed WSN-A Survey. International Journal of Grid and Distributed Computing, $8(6)$, pp.57-66.

[19] Yuvashree, A.K. and Venkatakrishnan, M., 2015. Survey on Range Free - Localization Schemes Based on Anchors Count in WSN Model. International Journal for Innovative Research in Science and Technology, 1(9), pp.186-192.

[20] Lee, J.Y., Yoon, C.H., Hyunjae, P. and So, J., 2013. Analysis of location estimation algorithms for wi fi fingerprint-based indoor localization. In Proceedings of the 2nd International Conference on Software Technology (Vol. 19, pp. 89-92).

[21] T. He, C. Huang \& B.M. Blum, J.A. Stankovic, T. Abdelzaher (2003) "Range-free localization schemes for large scale sensor networks", 9th annual international conference on mobile computing and networking, pp 81-95, San Diego.

[22] Mesmoudi, A., Feham, M. and Labraoui, N., 2013. Wireless sensor networks localization algorithms: a comprehensive survey. arXiv preprint arXiv:1312.4082.

[23] Denyuan Xu ,Benniu Zhang and Zhongwei Hou "Novel PIT Localization Algorithm based on Coverage of Anchors in WSN" JOURNAL OF NETWORKS, VOL. 7, NO. 9, SEPTEMBER 2012 
[24] WU Tie-zhou1, ZENG Yi-shi ,ZHOU Hui-jun, LI Biao "Wireless Sensor Network Node Location Based on Improved APIT" Journal of Surveying and Mapping Engineering Jun. 2013, Vol. 1 Iss. 1, PP. 15-19.

[25] Xiaofeng Li, Liangfeng Chen, Jianping Wang, Zhong Chu, Qiyue Li, Wei Sun Fuzzy system and Improved APIT (FIAPIT) combined range-free localization method for WSN KSII Transactions on Internet and Information Systems (TIIS). 2015. Jul, 9(7): 2414-2434 .

[26] Xingming Sun, Yachao Hu, Baowei, Wang, Jian Zhang, and Tao Li “VPIT: An Improved Range-Free Localization Algorithm Using Voronoi Diagrams for Wireless Sensor Networks" International Journal of Multimedia and Ubiquitous Engineering Vol.10, No.8 (2015), pp.23-34

[27] Adams, L. 1965. Progress in ecological biotelemetry. BioScience 15:83-86.

[28 ]Cohran, W. W., D. W. Warner, J. R. Tester, and V. B. Kuechele 1965. Automatic radio-tracking system for monitoring animal movements. BioScience 15:98-100.

[29] Nams, Vilis O., and Stan Boutin. "What is wrong with error polygons?." The Journal of wildlife management (1991): 172-176.

[30] Heezen, Keith L., and John R. Tester. "Evaluation of radio-tracking by triangulation with special reference to deer movements." The Journal of Wildlife Management (1967): 124-141.

[31] Montgomery A. Robert, Roloff J. Gary and Ver Hoeff M. Jay Implications of Ignoring Telemetry Error on Inference in Wildlife Journal of Wildlife Management, Vol. 75, No. 3 (April 2011), pp. $702-708$

[32] Ward, Michael P., Jinelle H. Sperry, and Patrick J.

Weatherhead. "Evaluation of automated radio telemetry for quantifying movements and home ranges of snakes." Journal of Herpetology 47.2 (2013): 337-345.

[33] D. Sharma, S. Verma, K. Sharma "Network Topologies in Wireless Sensor Networks: A Review" IJECT Vol. 4, Issue Spl - 3, April - June 2013.

[34] Nivedita Bisht Sapna Singh "ANALYTICAL STUDY OF DIFFERENT NETWORK TOPOLOGIES” International Research Journal of Engineering and Technology (IRJET) e-ISSN: 2395-0056 Volume: 02 Issue: 01 | Mar-2015.

[35] https://en.wikipedia.org/wiki/Mesh_networking

[36] Shamneesh Sharma, Dinesh Kumar and Keshav Kishore "Wireless Sensor Networks- A Review on Topologies and Node Architecture" International Journal of Computer Sciences and Engineering Vol.-1(2), pp (19-25) Oct 2013

[37] Jolly Soparia Nirav Bhatt, "'A Survey on Comparative Study of Wireless Sensor Network Topologies"' International Journal of Computer Applications (0975 - 8887) Volume 87 No.1, February 2014.
[38] D. Sharma, S. Verma, K. Sharma "Network Topologies in Wireless Sensor Networks: A Review IJECT Vol. 4, Issue Spl - 3, April - June 2013.

[39] Lambrou, T.P. Panayiotou, C.G., “A Survey on Routing Techniques Supporting Mobility in Sensor Networks", $5^{\text {th }}$ International Conference on Mobile Ad-hoc and Sensor Networks (MSN), pp. 78 - 85, 2009.

[40] S.Dewangan*, A.Kumar Pandey,N.Verma, D. Xaxa "A Comparative Assessment of Topologies and their issues in Wireless Sensor Networks" International Journal of Engineering Sciences \& Research Technology -ISSN: 2277-9655 May 2015.

[41] Chuan Zhu ,Chunlin Zheng LeiShu ,Guangjie Han "A survey on coverage and connectivity issues in wireless sensor networks" - Journal of Network and Computer Applications $\quad 35$ (2012) 619-632.

[42] C.-F. Huang, Y.-C. Tseng, The coverage problem in a wireless sensor network, in: ACM International Workshop on Wireless Sensor Networks and Applications, 2003, pp. 115-121.

[43] G. Xing, X. Wang, Y. Zhang, C. Lu, R. Pless, C. Gill, Integrated coverage and connectivity configuration for energy conservation in sensor networks, ACM Trans. Sensor Networks, in press.

[44] Li-Hsing Yen *, Chang Wu Yu, Yang-Min Cheng, "Expected k-coverage in wireless sensor networks" Ad Hoc Networks Volume 4, Issue 5, September 2006,Pages 636-650

[45] Ghosh, A. and Das, S.K., 2008. Coverage and connectivity issues in wireless sensor networks: A survey. Pervasive and Mobile Computing,4(3), pp.303-334.

[46] J. O'Rourke, Computational geometry column 15. Int'l Journal of Computational Geometry and Applications, Pp 215-217, 1992.

[47] S. Meguerdichian,F. Koushanfar, M. Potkonjak, and M.B.Srivastava. " Coverage problems in wireless ad-hoc sensor Networks". In IEEE INFOCOM, pp 1380-1387,

[48] F. Ye, G. Zhong, S. Lu, and L. Zhang, "PEAS: A robust energy conserving protocol for long-lived sensor networks". In Int'l Conf. on Distributed Computing Systems (ICDCS), 2003.

[49] Patra, R.R. and Patra, P.K., 2011. Analysis of k-coverage in wireless sensor networks Analysis, 2(9).

[50] Chuan Zhu, Chunlin Zheng LeiShu, Guangjie Han “A survey on coverage and connectivity issues in wireless sensor networks" - Journal of Network and Computer Applications 35 (2012) 619-632.

[51] Ashwini V. Nagpure Sulabha Patil "Topology Control in Wireless Sensor Network: An Overview” International 
Draft Version

Journal of Computer Applications (0975 - 8887) Volume 92 - No.7, April 2014.

[52] Rajat Bhardwaj and Hitesh Sharma, "Holes in Wireless Sensor Networks", Lovely Professional University, Phagwara, Punjab, India, hitesh.15778@1pu.co.in

[53] Jewel, D., Brundha, P., Wise, D.J.W. and Swaminathan, G.A., 2016. Improved Hole Detection Healing and Replacing Algorithm for Optimal Coverage in Wireless Sensor Networks.

[54] Ahmed, N., Kanhere, S.S. and Jha, S., 2005. The holes problem in wireless sensor networks: a survey. $A C M$ SIGMOBILE Mobile Computing and Communications Review, 9(2), pp.4-18.

[55] Bartariya, S. and Rastogi, A., 2016. Security in Wireless Sensor Networks: Attacks and Solutions. environment, $5(3)$.

[56] Gandhewar, N. and Patel, R., 2012, November. Detection and prevention of Sinkhole Attack on AODV protocol in Mobile Adhoc Network. In Computational Intelligence and Communication Networks (CICN), 2012 Fourth International Conference on (pp. 714-718). IEEE.

[57] Li, M. and Yang, B., 2006, June. A Survey on Topology issues in Wireless Sensor Network. In $I C W N$ (p. 503).

[58] Wang, W. and Bhargava, B., 2004, October. Visualization of wormholes in sensor networks. In Proceedings of the $3^{\text {rd }}$ ACM workshop on Wireless security (pp. 51-60). ACM. 